\title{
A NOTE ON PLANAR RANDOM MOTION AT FINITE SPEED
}

\author{
ALEXANDER D. KOLESNIK, ${ }^{*}$ Academy of Sciences of Moldova
}

\begin{abstract}
A simple derivation of the explicit form of the transition density of a planar random motion at finite speed, based on some specific properties of the wave propagation on the plane $\mathbb{R}^{2}$, is given.

Keywords: Random motion; finite speed; random evolution; explicit probability law; wave propagation; wave diffusion; wave equation; fundamental solution; Huygens principle
\end{abstract}

2000 Mathematics Subject Classification: Primary 60K99

Secondary 62G30; 60K35; 60J60; 60H30

This note is motivated by the previous works on planar random motions at finite speed by Stadje (1987), (1989), Masoliver et al. (1993), and Kolesnik and Orsingher (2005). In these works the following planar stochastic motion was studied. A particle starts from the origin of the plane $\mathbb{R}^{2}$, i.e. $x_{1}=x_{2}=0$, at time $t=0$ and moves with constant finite speed $c$. The initial direction is a two-dimensional random vector with uniform distribution on the unit circumference

$$
S_{1}=\left\{\boldsymbol{x}=\left(x_{1}, x_{2}\right) \in \mathbb{R}^{2}: x_{1}^{2}+x_{2}^{2}=1\right\} .
$$

The particle changes its direction at random instants, which form a homogeneous Poisson process of rate $\lambda>0$. At these moments it instantaneously takes on the new direction with uniform distribution on $S_{1}$, independently of its previous motion.

Let $\boldsymbol{X}(t)=\left(X_{1}(t), X_{2}(t)\right)$ denote the particle's position at an arbitrary time $t>0$. At any time $t>0$ the particle is located in the following planar disc of radius $c t$ :

$$
B_{c t}=\left\{\boldsymbol{x}=\left(x_{1}, x_{2}\right) \in \mathbb{R}^{2}: x_{1}^{2}+x_{2}^{2} \leq c^{2} t^{2}\right\} .
$$

The distribution $\operatorname{Pr}\{X(t) \in \mathrm{d} \boldsymbol{x}\}, \boldsymbol{x} \in B_{c t}, t \geq 0$, consists of two components. The singular component corresponds to the case when no Poisson event occurs in the interval $(0, t)$ and is concentrated on the circumference

$$
S_{c t}=\partial B_{c t}=\left\{\boldsymbol{x}=\left(x_{1}, x_{2}\right) \in \mathbb{R}^{2}: x_{1}^{2}+x_{2}^{2}=c^{2} t^{2}\right\} .
$$

For any $t>0$, the particle is located on $S_{c t}$ with probability

$$
\operatorname{Pr}\left\{\boldsymbol{X}(t) \in S_{c t}\right\}=\mathrm{e}^{-\lambda t} .
$$

If one or more than one Poisson event occurs, the particle lies strictly inside the disc $B_{c t}$, and the probability of this event is

$$
\operatorname{Pr}\left\{\boldsymbol{X}(t) \in \text { int } B_{c t}\right\}=1-\mathrm{e}^{-\lambda t}
$$

Received 19 January 2007; revision received 25 July 2007.

* Postal address: Institute of Mathematics and Computer Science, Academy of Sciences of Moldova, Academy Street 5 , Kishinev, MD-2028, Moldova. Email address: kolesnik@math.md 
The part of the distribution $\operatorname{Pr}\{\boldsymbol{X}(t) \in \mathrm{d} \boldsymbol{x}\}$ corresponding to this case is concentrated in the interior

$$
\text { int } B_{c t}=\left\{\boldsymbol{x}=\left(x_{1}, x_{2}\right) \in \mathbb{R}^{2}: x_{1}^{2}+x_{2}^{2}<c^{2} t^{2}\right\},
$$

and forms its absolutely continuous component. Therefore, there exists the density $p(x, t)=$ $p\left(x_{1}, x_{2}, t\right), \boldsymbol{x} \in$ int $B_{c t}, t>0$, of the absolutely continuous component of the distribution $\operatorname{Pr}\{\boldsymbol{X}(t) \in \mathrm{d} \boldsymbol{x}\}$. The reason for the existence of $p(\boldsymbol{x}, t)$ inside $B_{c t}$ is that it can be represented as a Poissonian sum of convolutions.

The principal known result states that the density $p(\boldsymbol{x}, t)$ has the following elegant form:

$$
\begin{gathered}
p(\boldsymbol{x}, t)=\frac{\lambda}{2 \pi c} \frac{\exp \left(-\lambda t+(\lambda / c) \sqrt{c^{2} t^{2}-\|\boldsymbol{x}\|^{2}}\right)}{\sqrt{c^{2} t^{2}-\|\boldsymbol{x}\|^{2}}}, \\
\boldsymbol{x} \in \operatorname{int} B_{c t}, \quad\|\boldsymbol{x}\|^{2}=x_{1}^{2}+x_{2}^{2}, \quad t>0
\end{gathered}
$$

The density, (2), was first obtained by Stadje (1987), (1989) using recursive arguments. Then it was derived by Masoliver et al. (1993) by means of finding and inverting the FourierLaplace transforms of the partial (with respect to directions) transition densities that satisfied some integral relation. Finally, the density was obtained by Kolesnik and Orsingher (2005) using characteristic function techniques. In all of these works the derivation of (2) involves nontrivial calculations and even requires a certain contrivance.

In this paper we give a simple derivation of the density, (2), based on some specific features of the wave propagation in the plane. Our goal is to demonstrate how easily this important result can be obtained from an appropriate physical interpretation of the model.

The relationships between finite-velocity random motions with continuous sample paths controlled by a Poisson process and wave processes have been well known for a long time. It was noted by many researchers that the density of such a finite-velocity random motion started at the origin represents a damped spatial wave that spreads outwards with finite speed. It is also well known that wave propagation has different features in different dimensions. In particular, in the even-dimensional spaces the wave diffusion takes place and the Huygens principle is not valid; see, for instance, Vladimirov (1981, Chapter 3, Section 14, Subsections 2, 3, 4).

In the planar case, the wave propagation with speed $c$ is described by the two-dimensional wave equation

$$
\frac{\partial^{2} u}{\partial t^{2}}=c^{2}\left(\frac{\partial^{2} u}{\partial x_{1}^{2}}+\frac{\partial^{2} u}{\partial x_{2}^{2}}\right)
$$

where $u=u(\boldsymbol{x}, t)=u\left(x_{1}, x_{2}, t\right), t>0$. The fundamental solution of this equation is given by

$$
u(\boldsymbol{x}, t)=\frac{1}{2 \pi c \sqrt{c^{2} t^{2}-\|\boldsymbol{x}\|^{2}}}, \quad\|\boldsymbol{x}\|<c t .
$$

From this formula it follows that the perturbation spreading from the point-like instant source $\delta(x) \delta(t)$ is concentrated (by the time $t>0$ ) in the closed disc of radius $c t$ centered at the origin. Thus, we can observe the forward front of the wave $\|\boldsymbol{x}\|=c t$, which moves on the plane with speed $c$. This perturbation is maintaining behind the forward wave front in all further time moments whereas the backward wave front is absent. 
We now assert that the transition density $p(\boldsymbol{x}, t)$ of the planar motion $\boldsymbol{X}(t)$ can be written in the form of the uniformly converging series, i.e.

$$
p(\boldsymbol{x}, t)=\mathrm{e}^{-\lambda t} \sum_{k=-1}^{\infty} a_{k}\left(\sqrt{c^{2} t^{2}-\|\boldsymbol{x}\|^{2}}\right)^{k},
$$

where the coefficients $a_{k}, k \geq-1$, do not depend on the spatial variable $\boldsymbol{x}$. Representation (5) is unique.

To justify (5), we first note that the exponential factor must be present in (5) owing to the fact that, since the motion is governed by a Poisson process of rate $\lambda$, the transition density $p(\boldsymbol{x}, t)$ can be written as follows:

$$
p(\boldsymbol{x}, t)=\mathrm{e}^{-\lambda t} \sum_{n=1}^{\infty} \frac{(\lambda t)^{n}}{n !} p_{n}(\boldsymbol{x}, t),
$$

where $p_{n}(\boldsymbol{x}, t)$ are the conditional densities subject to the event $\{N(t)=n\}$ and $N(t)$ denotes the number of Poisson events that have occurred during the time interval $(0, t)$. From (6) it follows that the exponential factor must be present in the transition density in any dimension. This fact emphasizes the damping effect of the motion.

Now we justify the form of the series in (5). As our process develops at finite velocity, it is described by some hyperbolic partial differential equation. As the speed of the motion is constant and the environment is homogeneous (such a type of motion is referred to as the isotropic transport process), it must be a second-order hyperbolic equation with constant coefficients. The transition density $p(\boldsymbol{x}, t)$ is the fundamental solution (Green's function) of this equation. It is well known (see, for instance, Courant and Gilbert (1962, Chapter 3, Section 3, Subsection 2)) that the fundamental solution (Green's function) of such a type of equation is expressed in terms of the 'hyperbolic distance'

$$
w(\boldsymbol{x}, t)=\sqrt{c^{2} t^{2}-\|\boldsymbol{x}\|^{2}}
$$

The function $w(\boldsymbol{x}, t)$ and its powers play a very important role in the study of isotropic processes. From the point of view of mathematical physics, the function $w^{2}(\boldsymbol{x}, t)$ is the characteristic cone of the wave equation, (3); see, for instance, Courant and Gilbert (1962, Chapter 6, Section 1, Subsection 2). The fundamental solution, (4), to the wave equation, (3), is also expressed as a power of (7), namely, $u(\boldsymbol{x}, t)=(1 /(2 \pi c)) w^{-1}(\boldsymbol{x}, t)$. This is because the function $w(\boldsymbol{x}, t)$ given by (7), as well as its integer powers, represent the traveling plane waves.

The classical method of plane waves is one of the most powerful tools in mathematical physics; see John (1955, Chapters 1, 2, and 4) or Courant and Gilbert (1962, Chapter 6, Sections 12,13, and 14). It is based on the fundamental wave superposition principle; see, for instance, Vladimirov (1981, Chapter 3, Section 14). According to this principle, every spatial wave can be expanded into a linear combination of the elementary plane waves and, inversely, any linear combination of the plane waves generates some spatial wave. Therefore, the density of our motion $p(\boldsymbol{x}, t)$ (which, we remind the reader, is some plane wave and, on the other hand, is a Green's function of some hyperbolic equation) can be represented in the form of a series with respect to the integer powers of the function $w(\boldsymbol{x}, t)$. However, since the function $p(\boldsymbol{x}, t)$ must be integrable (in the disc $B_{c t}$ ), we should take into account only integrable powers of the function $w(\boldsymbol{x}, t)$. 
By changing to polar coordinates we can easily show that the integrable powers of (7) are given by

$$
\begin{aligned}
\int_{B_{c t}}\left(\sqrt{c^{2} t^{2}-\|\boldsymbol{x}\|^{2}}\right)^{k} \mathrm{~d} \boldsymbol{x} & =\iint_{x_{1}^{2}+x_{2}^{2} \leq c^{2} t^{2}}\left(c^{2} t^{2}-\left(x_{1}^{2}+x_{2}^{2}\right)\right)^{k / 2} \mathrm{~d} x_{1} \mathrm{~d} x_{2} \\
& =2 \pi(c t)^{k+2} \int_{0}^{1} z\left(1-z^{2}\right)^{k / 2} \mathrm{~d} z \\
& =\frac{2 \pi(c t)^{k+2}}{k+2}, \quad k \geq-1 .
\end{aligned}
$$

Note that, for $k \leq-2$, the integral on the left-hand side of (8) does not exist. Therefore, we should consider only the powers $k \geq-1$ in (5).

To find the coefficients $a_{k},(5)$ is integrated over the disc $B_{c t}$. Then, taking into account (1) and (8), we have

$$
1-\mathrm{e}^{-\lambda t}=\mathrm{e}^{-\lambda t} \sum_{k=-1}^{\infty} a_{k} \frac{2 \pi(c t)^{k+2}}{k+2} .
$$

By multiplying this equality by $\mathrm{e}^{\lambda t}$ we obtain

$$
\mathrm{e}^{\lambda t}-1=\sum_{k=1}^{\infty} a_{k-2} \frac{2 \pi(c t)^{k}}{k} .
$$

By writing the Taylor expansion for the function on the left-hand side of this equality we can represent it in the following form:

$$
\sum_{k=1}^{\infty} \frac{\lambda^{k}}{k !} t^{k}=\sum_{k=1}^{\infty} a_{k-2} \frac{2 \pi c^{k}}{k} t^{k} .
$$

Equality (9) must be satisfied for any $t>0$. Therefore, it must be fulfilled identically. This means that both sides of (9) represent the Taylor expansions of the same smooth function of the variable $t$. However, the Taylor expansion of any smooth function is unique. Therefore, the coefficients of both series in (9) must coincide and we obtain

$$
\frac{\lambda^{k}}{k !}=a_{k-2} \frac{2 \pi c^{k}}{k}, \quad k \geq 1 .
$$

From this we find the desired coefficients

$$
a_{k}=\frac{\lambda^{k+2}}{2 \pi(k+1) ! c^{k+2}}, \quad k \geq-1 .
$$

Now, by substituting (10) into (5) we obtain

$$
\begin{aligned}
p(\boldsymbol{x}, t) & =\mathrm{e}^{-\lambda t} \sum_{k=-1}^{\infty} \frac{\lambda^{k+2}}{2 \pi(k+1) ! c^{k+2}}\left(\sqrt{c^{2} t^{2}-\|\boldsymbol{x}\|^{2}}\right)^{k} \\
& =\frac{\lambda}{2 \pi c} \frac{\mathrm{e}^{-\lambda t}}{\sqrt{c^{2} t^{2}-\|\boldsymbol{x}\|^{2}}} \sum_{k=0}^{\infty} \frac{1}{k !}\left(\frac{\lambda}{c} \sqrt{c^{2} t^{2}-\|\boldsymbol{x}\|^{2}}\right)^{k} \\
& =\frac{\lambda}{2 \pi c} \frac{\exp \left(-\lambda t+(\lambda / c) \sqrt{c^{2} t^{2}-\|\boldsymbol{x}\|^{2}}\right)}{\sqrt{c^{2} t^{2}-\|\boldsymbol{x}\|^{2}}}
\end{aligned}
$$

and this exactly coincides with (2). 
The uniqueness of (5) can be easily proved in the same manner.

Remark 1. The density, (2), can be rewritten as

$$
p(\boldsymbol{x}, t)=\frac{\lambda}{2 \pi c \sqrt{c^{2} t^{2}-\|\boldsymbol{x}\|^{2}}} \exp \left(-\lambda t+\frac{\lambda}{c} \sqrt{c^{2} t^{2}-\|\boldsymbol{x}\|^{2}}\right) .
$$

It is interesting to note that the first factor of this function is exactly (up to the constant $\lambda$ ) the fundamental solution, (4), to the two-dimensional wave equation, (3), whereas the second (exponential) factor introduces the damping effect.

Remark 2. In our analysis the specific properties of the wave propagation on the plane $\mathbb{R}^{2}$ have substantially been used. In particular, the well-known idea of mathematical physics to represent a planar wave in the form of a combination of elementary plane waves has been applied in writing (5). However, in other dimensions this idea, apparently, cannot be used directly. In particular, in the odd-dimensional cases the Huygens principle is valid and the wave diffusion does not take place. Therefore, it seems unlikely that the transition density can be written in the form of (5). In the higher even-dimensional cases this idea can, apparently, be used; however, an expansion with respect to the spherical (Legendre) functions should be considered.

Remark 3. The fact that our motion is controlled by a homogeneous Poisson process plays a crucial role in our analysis. In this case the transition density $p(\boldsymbol{x}, t)$ behaves like some plane wave (a fact that has been noted by many researchers). If the governing process is not Poissonian (i.e. the random times between turns are not exponentially distributed), we cannot assert that the density has the form of some plane wave (to the best of our knowledge, such results have not been obtained so far). Therefore, our analysis is not applicable in this case.

\section{Acknowledgement}

The author wishes to thank an anonymous referee for insightful comments concerning the representation of the density, (5).

\section{References}

Courant, R. and Gilbert, D. (1962). Methods of Mathematical Physics, Vol 2, Partial Differential Equations. Interscience, New York.

John, F. (1955). Plane Waves and Spherical Means Applied to Partial Differential Equations. Interscience, New York.

KolesniK, A. D. AND Orsingher, E. (2005). A planar random motion with an infinite number of directions controlled by the damped wave equation. J. Appl. Prob. 42, 1168-1182.

Masoliver, J., Porrá, J. M. And Weiss, G. H. (1993). Some two and three-dimensional persistent random walks. Physica A 193, 469-482.

Stadje, W. (1987). The exact probability distribution of a two-dimensional random walk. J. Statist. Phys. 46, $207-216$. Stadje, W. (1989). Exact probability distributions for non-correlated random walk models. J. Statist. Phys. 56, $415-435$. Vladimirov, V. S. (1981). The Equations of Mathematical Physics. Nauka, Moscow. 